\title{
Die Predigt aus theologischer Sicht
}

Obwohl die vorliegende Arbeit im Bereich der (multimodalen) Interaktionsanalyse verortet ist, und daher die in Kapitel 4 und 5 vorgestellten Forschungslinien zentral für die nachfolgende Analyse sind, ist es fruchtbar, sich dem Gegenstand der Predigt auch aus theologischer bzw. homiletischer Perspektive anzunähern. Ein spezielles Interesse gilt dabei Ansätzen, die sich explizit auf linguistische Theorien stützen. Aufgrund der fachlichen Ausrichtung dieser Arbeit sollen die einzelnen homiletischen Perspektiven an dieser Stelle jedoch nur grob umrissen werden. Weitere Ausführungen und Konkretisierungen folgen in den spezifischeren Analysekapiteln dieser Arbeit.

Während sich innerhalb der linguistischen und soziologischen Forschungstradition nur wenige Studien explizit mit dem Gegenstand der Predigt auseinandergesetzt haben, hat sich innerhalb der (Praktischen) Theologie die Homiletik als ein eigenes Teilgebiet herausgebildet (siehe zu den verschiedenen Bereichen der Praktischen Theologie Nicol 2000, Möller 2004, Wollbold 2017:70-75). Als Lehre von der Predigt (vgl. Nicol/Deeg 2012:68) bzw. ,Metakommunikation kirchlicher Rede“" (Thiele 2004:42) setzt sich die Homiletik mit unterschiedlichen Predigtformen auseinander und fragt synchron und diachron nach Herausforderungen, Grenzen, Möglichkeiten und Entwicklungen der Predigt (vgl. Zerfaß 2002:1349) sowie nach ihrer gegenwartsadäquaten und rezipientenorientierten ästhetischkommunikativen Gestaltung (vgl. Beutel/Drehsen 1999:7). Die Homiletik rückt ihrem Selbstverständnis entsprechend vor allem die Frage nach den charakteristischen Merkmalen und der praktischen Realisierung einer Predigt in den Fokus ihrer Forschung (vgl. Thiele 2004:42, Wollbold 2017). Die essentielle Frage, was eigentlich eine Predigt sei und was sie ausmache, steht konfessionenübergreifend am Anfang und im Mittelpunkt zahlreicher homiletischer Arbeiten und praktisch orientierter Handreichungen und Arbeitsbücher (vgl. Wollbold 2017:62) und gehöre nachgerade zum „Kerngeschäft der Homiletik“ (Lütze 2006:296). So 
oft die Frage nach den konstitutiven Merkmalen jedoch gestellt wird, so oft wird betont, dass sie sich (wissenschaftlich) nicht in einem Satz und darüber hinaus vielleicht nicht einmal ansatzweise adäquat beantworten lasse und die Fülle der Definitionen entsprechend groß sei (vgl. Nembach 2002:123-131, Thiele 2004, Lütze 2006:296 f., Wollbold 2017:62).

Etymologisch bezeichnet der von lateinisch ,predica“ entlehnte Begriff ,Predigt' zunächst einen öffentlichen Vortrag und das entsprechende Verb ,predigen“ (von lat. ,praedicare') etwas (mündlich) öffentlich bekannt zu machen, zu verkünden, allgemeiner: laut zu sagen (vgl. Kluge 2002:719, Zerfaß 2002:1348). Bis heute wird die Predigt in der Homiletik übereinstimmend in ihrem Charakter als Rede reflektiert (vgl. u. a. Schütz 1981:37, Daiber 1991:15, Nembach 2002:70, Thiele 2004:30, Wollbold 2017:34). Da diese Bestimmung jedoch nicht hinreichend ist und andere Gattungen (wie die politische Rede) ebenfalls das Merkmal der öffentlichen Rede tragen, wird die Predigt zudem hinsichtlich institutioneller, räumlicher, inhaltlicher und kommunikativer Kriterien bestimmt: Die Predigt wird als kirchliche bzw. gottesdienstliche und damit institutionell gerahmte Rede, mithin als „,kirchliche Auftragsrede“ (Brinkmann 2000:140) definiert (vgl. u. a. Daiber 1991:15, Nembach 2002:70, Zerfaß 2002:1348, Thiele 2004:42, Meyer 2014:1, Wollbold 2017:17). Zerfaß sieht den Terminus der Predigt gar als Oberund Leitbegriff kirchlich autorisierter Verkündigung und verweist in diesem Zusammenhang darauf, dass die Verkündigung von Gottes Wort nicht ausschließlich der Predigt obliegt (vgl. Zerfaß 2002:1348; siehe Kapitel 13). Der Raum ist insofern relevant, als dass die Predigt von einem speziellen Ort, nämlich „traditionell von der Kanzel aus gehalten" (Nembach 2002:71) wird (siehe Kapitel 14). Die Predigt ist inhaltlich nicht nur eine Rede, sondern eine „,christlich-religiöse Rede“ (Daiber 1991:199), die die Aufgabe der „Kommunikation über das Evangelium“ (Thiele 2004:29, siehe auch Daiber 1991:207) bzw. die „Kommunikation des Evangeliums“ (Engemann 2002:138; siehe auch Mette 2005:11) habe. In diesem Zusammenhang entsteht auch die Diskussion darüber, ob die Tradierung, Auslegung und Auseinandersetzung mit dem Wort Gottes selbst Wort Gottes und Heilsgeschehen ist oder nicht (vgl. Schütz 1972:7; siehe Kapitel 16). Die Predigt unterliegt zudem bestimmten Kommunikationsbedingungen, etwa einer spezifischen Interaktionsordnung (,öffentliche Rede face-to-face“ (Wollbold 2017:17; siehe Kapitel 13 und 14). Als Konsequenz aus diesem Befund werden monologische und dialogische Strukturen betrachtet (siehe Kapitel 17) und die mediale Beschaffenheit in den Blick genommen.

Die Entstehungsgeschichte der Gattung ,Predigt" reicht zurück bis in vorliterale Zeiten und gründet sich in jüdisch-synagogalen Vermittlungstraditionen (vgl. 
Schütz 1972:6, Engemann 2002:87 f.), bei denen es bis heute neben der Traditionsbewahrung auch um die Auslegung der gelesenen Schrift und damit um theologische Lehrtätigkeiten geht (vgl. Daiber 1991:37, Elbogen 1995:194). Die jüdische Predigt hat jedoch im Laufe ihrer Geschichte Wandlungen erfahren und wurde von einer reinen Lehrpredigt zu einer erbaulichen Predigt, in der auf der Grundlage der vorgelesenen Schrifttexte die behandelten Themen frei entfalten werden (vgl. Elbogen 1995:196). Für die christliche Predigt im Allgemeinen hat diese jüdische Traditionslinie u. a. die Auseinandersetzung mit biblischen Texten und religiösen Gesetzen sowie einer Anleitung zur „Umsetzung im Alltag“ (Engemann 2002:88) zur Folge. Daraus ergeben sich bis heute Charakterisierungen der Predigt als mahnende, moralische, aber auch erbauliche Rede. Anleihen an jüdische Traditionen werden darüber hinaus in der Bibelexegese, d. h. in der Auslegung der Texte, gesehen (vgl. Engemann 2002:89).

Die Predigt ist seit den Anfängen der Institution Kirche ein fester Bestandteil des christlichen Gottesdienstes (siehe Kapitel 13) und gehört neben den Textlesungen und den Fürbittgebeten zu den ältesten und bis heute praktizierten Formen christlicher Frömmigkeit (vgl. Schütz 1972:8). Dabei zeigt sich ein beständiger inner- und interkonfessioneller Wandel des Predigtverständnisses (vgl. Daiber 1991:37). Vor allem mit der Reformation hat eine grundlegende Verschiebung hinsichtlich der Position und der Wertigkeit der Predigt in der christlichen und vor allem der evangelischen und reformierten Gottesdienstlandschaft stattgefunden: „Das zentrale Geschehen des Gottesdienstes ist nicht mehr das Mahl, sondern das anredende Wort der Predigt“ (Daiber 1991:38; zum Stellenwert der Predigt in den Konfessionen siehe Kapitel 13). Vor diesem Hintergrund der Aufwertung der Predigt im evangelisch-reformierten Gottesdienst wird zunehmend auch das Performanzgeschehen ,Predigt ${ }^{*}$ thematisiert. Gleichzeitig ist der Begriff der Predigt ein alltäglich gebrauchter Terminus, der als Ethnokategorie sowohl von der Gemeinde als auch von der Öffentlichkeit verwendet wird und dem zahlreiche alternative Begriffe wie ,Botschaft', ,Mitteilung' oder ,Verkündigung ' an die Seite gestellt werden (vgl. Nembach 2002:70 f.). Zudem werden sprachlich-stilistische Ausdrucksformen (anderer) literarischer Gattungen und alltagssprachliche Elemente in die Predigt übernommen (vgl. Belke 1973:121, Paul 2009:2258). Die Predigt als „geistliche Form der Rede“ (Belke 1973:120) steht somit in einer engen Beziehung und in einem Spannungsverhältnis zu nichtinstitutionellen Kommunikations- und Interaktionsereignissen. In diesem Zusammenhang wird auch die Diskussion um die Funktion, der Legitimation sowie der Qualität zeitgenössischer christlicher Predigten geführt. Dies bedingt die Herausbildung zweier Lager innerhalb der theologischen Forschung. 
Während die eine Seite die grundlegende gesellschaftlich und biblisch begründbare Notwendigkeit der Predigt betont (vgl. Klippert 2008:19, Wollbold 2017:16) und Reformen anzuregen bestrebt ist (vgl. Eickhoff 2009), fragt die andere Seite, ob die Form der Predigt überhaupt noch zeitgemäß ist und Verkündigung nicht auch ohne sie geschehen kann (siehe zu dieser Diskussion die Darstellungen in Plüss 2018). Als wichtiges Argument wird dann eine nach wie vor bestehende Predigtkrise postuliert (vgl. u. a. Güttgemanns 1972:7, Otto 1976:10, Schütz 1981:21, Baldwin 1984:2, Eickhoff 2009, Paul 2009:2268, Meyer 2014:5). Kritisiert werden neben der theologischen Tiefe oder der Bibeltreue der Ausführungen vor allem die Rhetorik, d. h. der Sprachgebrauch, die Art und Weise, wie die Prediger die gottesdienstliche Rede vortragen, und darüber hinaus, wie eine Vermittlung zwischen Bibeltext und Gegenwart geschieht (vgl. Flügge 2016:9; siehe Kapitel 16). Zentrale Schlagworte sind dabei: Aktualität, Hörernähe, Verständlichkeit, Anschaulichkeit und Authentizität (vgl. Flügge 2016:12 ff.; siehe auch Schütz 1981:26, Daiber 1991:207). Besonders die sich verändernden gesellschaftlichen und kommunikativen Bedingungen und deren Einfluss auf die Gestaltung von Predigten ist ein permanent aktualisiertes Problemfeld homiletischer Forschung (vgl. Engemann 2002:108, Thiele 2004:29, Vogt 2009:11), bei dem es um die Frage geht, wie Verkündigung im 21. Jahrhundert theologisch, rhetorisch und performativ gelingen kann und was eine ,gute', zeitgemäße Predigt ausmacht (vgl. z. B. Daiber 1991, Engemann 2002, Nembach 2002:94, Schirmer 2013:9). Gerade aus der Sichtweise einer rhetorischen Krise, d. h. einer vermeintlichen Krise der Performativität des Predigtvortrags heraus, wurde seit den 60er und 70er Jahren des 20.Jhd. immer wieder der Ruf nach grundlegenden Reformen der Predigtpraxis laut (vgl. u. a. Daiber et al. 1980, Luther 1983, Daiber 1991:201). Dies hat zum einen zur Entwicklung neuer Predigtmodelle und zum anderen zu einer ,empirischen Wende“ (vgl. Nicol 2005:14) geführt, in deren Zuge sich die Homiletik immer wieder anderen Wissenschaftsdisziplinen zugewandt und den Versuch unternommen hat, Konzepte, Modelle und Theorien aus der Sprachphilosophie, der Kommunikationswissenschaft, der Soziologie, der Linguistik sowie der Theaterwissenschaft fruchtbringend zu adaptieren (siehe u. a. Roth 2011 und die Übersicht in Meyer 2014:234 ff.). Thiele fragt allerdings, ob die vermeintlich auf sprachlicher Ebene anzusetzende Predigtkrise tatsächlich den Kern des Problems trifft, oder nicht vielmehr ein tieferes Legitimationsproblem der Homiletik hinter der seit Jahrzehnten aktualisierten Klage zur Predigtnot liege. Denn ohne Krise, keine Notwendigkeit einer Disziplin, die sich explizit mit der Predigt auseinandersetzt, so die spitze Kritik von Thiele (Thiele 2004:37 f.). 
Das zunehmende Bewusstsein für die Bedeutung von Sprache führte in der Theologie konfessionsübergreifend zu einer, linguistischen Wende‘. Die Linguistik wird im Zuge dieser Perspektivverschiebung zu einer neuen Bezugsdisziplin homiletischer Forschungen. Güttgemanns versteht die Theologie entsprechend als „textbezogene Wissenschaft“ (1972:7) und als „Wissenschaft der Rede von Gott“ (Güttgemanns 1972:7), deren zentraler Gegenstand mündlich und schriftlich geäußerte Texte sind. Die linguistische Theologie soll in ihrer Reflexion und Rezeption linguistischer Theorien und Methoden dem Zusammenhang zwischen Religion und Sprache bzw. Sprachgebrauch und der Frage danach, welche Elemente die Predigt zu einer Form religiöser Kommunikation machen, Rechnung tragen. Seiner Meinung nach kann nur eine systematische Reflexion des Verhältnisses zwischen Sprache und Theologie zu einer Überwindung der Kritik an der Predigt und der postulierten Predigtkrise führen. Damit bildet die linguistische Theologie das Pendent zur Theolinguistik (siehe Kapitel 4). Die Zusammenarbeit der beiden Richtungen beschränkt sich jedoch vorwiegend auf die Reflexion textlinguistischer Theorien und Modelle. Darüber hinaus wurden in der homiletischen Forschung vor allem die sprachphilosophischen Überlegungen Wittgensteins (vgl. Clausen 2010) und die Sprechakttheorie nach Austin und Searle rezipiert (vgl. Engemann 2002:137 ff., Lütze 2006, Vogt 2009, Arens 2009). So beschreibt Arens ,religiöse Sprachhandlungsvollzüge“ (2009:45) und bestimmt die Predigt als institutionellen Sprechakt mit „konstativen, regulativen und expressiven Elementen" (Arens 2009:49). Dabei bleibt jedoch weitgehend unreflektiert, dass es sich bei der Sprechakttheorie um ein sprachphilosophisches Konzept handelt, das die Forderung der in den 1970er Jahren aufkommenden Pragmatik nach empirischer Arbeit mit natürlichen Daten nur unzureichend einlöst. Bis heute wird die Sprechakttheorie für ihre Arbeit mit konstruierten Beispielen, ihr deduktives, sprecherzentriertes und empiriefernes Vorgehen kritisiert (vgl. Levinson 2000, Meier et al. 2019, Staffeldt 2019). Forschungstraditionen wie die Ethnographie der Kommunikation und die (ethnomethodologische) Konversationsanalyse mit ihren Weiterentwicklungen (siehe Kapitel 10) haben die Sprechakttheorie sowohl methodisch als auch theoretisch überholt (vgl. Levinson 2000:303). Jedoch reflektieren nach wie vor nur vereinzelte homiletische Studien diese linguistischen und soziologischen Forschungstraditionen. Im Zentrum der wenigen empirischqualitativen Analysen stehen dabei zum einen die Gesamtschau des Gottesdienstes und zum anderen visuell wahrnehmbare Interaktionseinheiten wie Gebete. Meyer verweist in seiner Studie über deutsche und amerikanische protestantische Predigten zwar auf Überlegungen der Ethnomethodologie und der Konversationsanalyse (vgl. Meyer 2014:246-248), und stellt darüber hinaus die Ethnographie der Kommunikation nach Dell Hymes vor (vgl. Meyer 2014:148-251), nutzt in seinen 
Analysen jedoch vor allem Interviews und die Befunde teilnehmender Beobachtung. Zudem geht Meyer bei seinen Beschreibungen von einem Interaktionsbegriff aus, der unter sprachlicher Praxis ein intentionales Geschehen versteht (vgl. Meyer 2014:238) und reflektiert nicht, dass es Ansätzen wie der Konversationsanalyse um die induktiv-datenbasierte Analyse und Beschreibung der situativ lokalen wechselseitigen Hervorbringung von Interaktion geht (siehe Kapitel 10). Methodisch richtet er seine Studie an der Grounded Theory und einem konstitutionsanalytischen Vorgehen aus (vgl. Meyer 2014:285). Besonders auffällig ist, dass Meyer zwar davon spricht, dass er die untersuchten Predigten audiovisuell erhoben habe, diese jedoch weder in Form von Screenshots noch als Transkripte in der Arbeit sichtbar werden. Auch Schirr stützt seine Studie zu Führbitten vor allem auf Ergebnisse teilnehmender Beobachtung, dichter Beschreibung sowie Interviews (vgl. Schirr 2018:87). Darüber hinaus stellt er die erhobenen Audioaufnahmen der untersuchten Gottesdienste und Fürbitten explizit nicht in den Fokus der Untersuchung (Schirr 2018:87). Lediglich Walti (2016) verfolgt in seiner Untersuchung agendenfreier Gottesdienste in reformierten Kirchen der Deutschschweiz einen konsequent empirisch-qualitativen, videoanalytischen Zugang. Aus theologischer Perspektive fragt er mithilfe (mikro)soziologischer Theorien und Methoden nach den ,impliziten Regeln [denen] die Gottesdienstinteraktion in reformierten Gottesdiensten folgt“" (Walti 2016:25). Mithilfe der Begriffe der Interaktionsordnung nach Goffman, der Ritualität nach Durkheim und Collins sowie der Interaktionsarchitektur nach Hausendorf/Schmitt (siehe Kapitel 14) versteht er Gottesdienste als interaktives, ritualisiertes Geschehen, das die Beteiligten aktiv mitgestalten (vgl. Walti 2016:41). Gegenstand seiner Betrachtung ist u. a. die verbale und visuelle Eröffnung der untersuchten Gottesdienste. Dabei arbeitet er die Pfarrpersonen als rituelle Protagonisten heraus, die im Verlauf der Eröffnung mithilfe verbaler und visueller Kontextualisierungshinweise (siehe Kapitel 10) zu Fokuspersonen werden (vgl. Walti 2016:123). Des Weiteren betrachtet Walti Gebete, Lesungen und das Abendmahl unter raumsoziologischen und interaktiven Aspekten, von denen er im Anschluss daran normativ-theologische Konsequenzen ableitet. Die Predigt wird zwar als charakteristisches Merkmal der untersuchten Gottesdienste erwähnt (vgl. Walti 2016:91 und 330), jedoch nicht untersucht und beschrieben.

Mithilfe theaterwissenschaftlicher Forschung soll u. a. die Frage nach den performativen Gestaltungsmöglichkeiten der Predigt beantwortet werden (vgl. u. a. Staub 2002). In diesem Zusammenhang haben sich drei Betrachtungslinien herausgebildet: a) die Auffassung der Predigt als Handwerk, d. h. als zu erlernende Kompetenz, b) die Auffassung der Predigt als zu übende und zu reflektierende Praxis, die dennoch auf den Fähigkeiten und dem persönlichen Stil des Predigers 
sowie dem Epochenstil der jeweiligen Zeit beruht (Predigt als Kunst-Handwerk vgl. Fechtner 2008:152; vgl. dazu auch Wollbold 2017) und c) die Auffassung der Predigt als Kunstwerk, die die Begabung des Predigers in den Vordergrund stellt (vgl. Wollbold 2017:65). Die dramaturgische Homiletik betrachtet die Predigt aus der ,Kunstwerk-Perspektive' heraus als Gesamtkunstwerk. Damit verschiebt sich gleichzeitig das Analyseinteresse vom Prediger hin zu den Predigthörenden. Diese werden nun als aktiv an der Entstehung der Predigt als Kunstwerk beteiligte Ko-Autoren angesehen (vgl. u. a. Garhammer 1998 und 2000, Thiele 2010, Lorensen 2011:27, Martin 2012, Nicol/Deeg 2012:68; siehe Kapitel 17). Damit folgt diese Theorie der Kunstwerktheorie nach Eco (vgl. Eco 1973), der im Rezipienten nicht mehr nur eine aufnehmende Instanz sieht, die den Text allein aus dem Text selbst heraus interpretiert und versteht, sondern als ein schöpfendes und erschaffendes Wesen, das den Sinn eines Textes aktiv herstellt, indem es das, was der Text liefert, mit individuell vorhandenen Wissensbeständen und Erfahrungen in Beziehung setzt (vgl. Thiele 2010:69). Die Predigt erscheint dabei als unabgeschlossener Text, der erst durch die Rezipierenden und deren individuelle und kollektiv geteilte Wissensbestände Vervollständigung erführt (vgl. Thiele 2004:31). Es ist die Aufgabe des Predigers, diesen Prozess des Verstehens zu berücksichtigen und in diesem Zusammenhang bereits in der Vorbereitung zur Predigt rezipientenorientiert zu arbeiten (vgl. Thiele 2010:70). Aus der stärkeren Berücksichtigung der Rezipierenden entwickelte Modelle sind die Konzepte des Bibliodramas und des Bibliologs, bei dem die Gottesdienstbesucher biblische Texte nachvollziehen, indem sie sich unter verschiedenen Fragestellungen, geführt durch den Prediger, in die Figuren der Texte hineinversetzen, d. h. in ihrer ,Vorstellung zu einer Gestalt aus der biblischen Geschichte werde" (Pohl-Patalong 2005:24).

Zahlreiche Studien fragen dann nach der Wirkungsästhetik von Predigten, den Rezeptionsbedingungen und den Höreindrücken der Anwesenden (vgl. Binder et al. 1973, Daiber et al. 1980, Daiber 1991, Lütze 2006, Schwier/Gall 2008; siehe dazu auch die Übersicht in Wollbold 2017:20 ff.). Arbeiten, die im Zuge dieser theoretischen und vor allem auch methodischen Ausrichtung entstanden, arbeiten z. B. mit Fragebögen, die der Gemeinde im Anschluss an den Gottesdienst ausgehändigt werden, mit Echtzeitmessungen emotionaler Beteiligung sowie mit Interviews, die mit Predigern oder Personen der Gemeinde geführt wurden (siehe dazu die Übersicht in Wollbold 2017:20 ff.). Aufbauend auf diesen wirkungsästhetischen Befunden haben sich wiederum zahlreiche praktische Handreichungen, Arbeitsbücher und Ratgeber mit der formalen Gestalt der Predigt auseinandergesetzt, die jedoch in ihrer Darstellung der herausgearbeiteten Kriterien und Anforderungen stark normativ bleiben und an Idealfällen orientiert 
sind (vgl. u. a. Härtner/Eschmann 2008, Boyd-MacMillan 2011, Keller 2017 und viele andere).

Darüber hinaus werden im Zuge der performativen Perspektivierung der Predigt und der Rezeption theaterwissenschaftlicher Forschungsansätze nichtsprachliche Elemente betrachtet. Studien, die sich mit der Körpersprache auseinandersetzen, vertreten dabei meist ein sehr breites Verständnis von visueller Kommunikation und schließen in ihre Betrachtungen neben mimischen und gestischen Komponenten auch die Kleidung mit ein. Zudem werden gustatorischer und olfaktorischer Elemente berücksichtigt (vgl. Wenz 1996, Thiele 2004:271 ff.). Dabei werden zwar die großen performativen Gesten - etwa das Zeigen von Hostie und Kelch - thematisiert (vgl. Wenz 1996), die kleinen visuellen Formen der Kommunikation und Koordination zur Herstellung und Durchführung des Interaktionsereignisses Gottesdienst bzw. Predigt bleiben dabei unterrepräsentiert. Gerade sie sind jedoch zentral, wenn es um die Frage nach der Etablierung der Predigt geht (siehe Kapitel 15).

Innerhalb der empirischen Forschung der Homiletik existieren also auf der einen Seite Studien, die mit Videodaten arbeiten, den Fokus ihrer Forschung allerdings nicht auf die Predigt legen, und auf der anderen Seite Arbeiten, die sich zwar dezidiert mit der Predigt auseinandersetzen, aber nicht die interaktive Struktur und situative multimodale Realisierung auf der Grundlage authentischer Daten in den Blick nehmen. Beide Ansätze zusammenzubringen ist also ein vorhandenes Desiderat homiletischer Forschung. Dies verwundert, da innerhalb des theologischen Studiums bzw. der homiletischen Ausbildung vielerorts mit Videoaufzeichnungen zu Schulungszwecken gearbeitet wird. Diese Daten wurden bislang jedoch nicht als gewinnbringende Grundlage für wissenschaftliche Auseinandersetzungen erkannt.

Die Menge der vorhandenen theologischen Arbeiten zur Predigt und die Auseinandersetzung mit Sprache und religiöser Kommunikation, täuscht darüber hinweg, dass nach wie vor nicht letztgültig geklärt ist, was eine Predigt gerade auf sprachlicher und interaktiver Ebene ausmacht und welche Elemente für sie konstitutiv sind. So thematisiert der Großteil der homiletischen Literatur, ,was eine Predigt zu einer Predigt machen sollte" (Lütze 2006:296), und verpasst es zu fragen, „was eine Predigt tatsächlich ist, d. h. welche Merkmale als typisch für das Genre Predigt gelten können“ (Lütze 2006:296; siehe dazu auch Beutel/ Drehsen 1999:7). Lütze kommt zu dem Ergebnis, dass „,[d]ie Erfassung der Textsorte Predigt anhand tatsächlicher Predigten [...] sowohl aus homiletischer als auch aus linguistischer Perspektive ein bislang kaum eingelöstes Desiderat dar[stellt]" (Lütze 2006:296). Neben theoretische und inhaltlich-normative Studien müssen also mehr empirisch qualitative Arbeiten treten, die anhand 
authentischer Daten das Predigtereignis auf seine Charakteristik hin untersuchen und differenziert beschreiben (vgl. dazu auch Heuser et al. 2013:11 und Malmström 2015:268 f.). Bei diesem Forschungsdesiderat setzt die vorliegende Arbeit an. Sie verknüpft den Gegenstand der christlichen Predigt mit methodischen und theoretischen Traditionslinien der Linguistik und der Soziologie, die in dieser Form in der homiletischen Forschung noch nicht zur Anwendung gebracht wurden. Die nachfolgenden Analysen zeigen jedoch, wie fruchtbar dies sein kann, um einen genaueren und authentischen Blick auf religiöse Ereignisse zu gewinnen.

Open Access Dieses Kapitel wird unter der Creative Commons Namensnennung 4.0 International Lizenz (http://creativecommons.org/licenses/by/4.0/deed.de) veröffentlicht, welche die Nutzung, Vervielfältigung, Bearbeitung, Verbreitung und Wiedergabe in jeglichem Medium und Format erlaubt, sofern Sie den/die ursprünglichen Autor(en) und die Quelle ordnungsgemäß nennen, einen Link zur Creative Commons Lizenz beifügen und angeben, ob Änderungen vorgenommen wurden.

Die in diesem Kapitel enthaltenen Bilder und sonstiges Drittmaterial unterliegen ebenfalls der genannten Creative Commons Lizenz, sofern sich aus der Abbildungslegende nichts anderes ergibt. Sofern das betreffende Material nicht unter der genannten Creative Commons Lizenz steht und die betreffende Handlung nicht nach gesetzlichen Vorschriften erlaubt ist, ist für die oben aufgeführten Weiterverwendungen des Materials die Einwilligung des jeweiligen Rechteinhabers einzuholen.

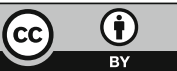

